\title{
SOME QUALITATIVE RESULTS IN THE LINEAR THEORY OF MICROPOLAR SOLID-SOLID MIXTURES
}

\author{
Ionel-Dumitrel Ghiba ${ }^{\dagger, \ddagger}$ and Cătălin Galeş \\ $\dagger$ "Alexandru Ioan Cuza" University of Iaşi, Department of Mathematics, \\ Blvd. Carol I, no.11, 700506- Iaşi, Romania \\ ‡ "Octav Mayer" Institute of Mathematics, Romanian Academy, \\ Blvd. Carol I, nr. 8, 700505-Iaşi, Romania \\ E-mail: dumitrel.ghiba@uaic.ro, cgales@uaic.ro
}

\begin{abstract}
This paper deals with the linear theory of micropolar solid-solid mixtures. First, some existence, uniqueness and continuous dependence results are derived. Then, the propagation of plane waves is studied. There are possible four types of waves: longitudinal displacement waves, longitudinal microrotation waves, transverse displacement waves and transverse microrotation waves. A detailed analysis is presented for the longitudinal displacement waves and the longitudinal microrotation waves.
\end{abstract}

Keywords: micropolar mixtures; solid-solid interaction; existence; waves

\section{INTRODUCTION}

Many natural or synthetic materials are not pure materials, but represent mixtures consisting of several constituents (solids, fluids or gases) having their own physical and chemical properties. Sometimes the presence of a constituent can be ignored, provided that one constituent prevails, but in many situations the local mechanical effects of each ingredient of the mixture cannot be disregarded. The classical theories cannot describe the thermomechanical behaviour of interacting continua. This is the reason for what various mixture theories, developed in Eulerian description or Lagrangian description, have been proposed in the last decades. The progress in the field can be found in the review articles by Bowen [1], Bedford and Drumheller [2] and the book of Rajagopal and Tao [3].

A number of papers (see for instance $[4,5,6,7,8,9,10]$ and the works cited therein) pay a special attention to include some terms in the basic formulation of mixture theory in order to reflect the microstructure of each constituent. Twiss and Eringen [4,5] have introduced the mixture theory of materials with microstructure. Two papers of Eringen [6,7] got back in discussion the study of micropolar mixtures. In these theories, each particle can independently translate and rotate, so that it has six degrees of freedom. The theories developed by Twiss and Eringen $[4,5]$ are enough to have a complete Eulerian description of the micropolar mixtures.

The Eulerian description is appropriated to study the behaviour of mixtures of fluids, while for the theory of mixtures of solids is naturally to use the Lagrangian description $[11,12]$. These two descriptions lead to different equations and to different results. Moreover, when the Lagrangian description is used to develop a theory of mixtures for solids, then the initial boundary value problems associated are formulated in a natural way and the boundary conditions have a clear physical and mathematical meaning.

In this paper we consider the linear theory of heat-conducting micropolar solid-solid mixtures [9] (see also $[8,10])$. This theory was derived by using the Lagrangian description. Our first goal is to establish some existence and uniqueness results for the solutions of the problems associated with both the equilibrium theory and the dynamic theory. For the later case, the paper also investigates the continuous dependence of solution with respect to the initial data and the body loads. These qualitative results prove that the approach of the linear theory derived in [9] is well posed.

In the final section we study the propagation of plane waves through an homogeneous and isotropic binary mixture. For such mixtures, the plane waves may be categorized as longitudinal waves and transverse waves, 
each of which can be split into displacement waves and microrotation waves. We find that the longitudinal waves and the transverse waves are separable. Moreover, since the longitudinal displacement waves and the longitudinal microrotation waves are also separable, a detailed analysis is given for these waves. Thus, in the case of longitudinal displacement waves we compute the speeds of propagation and discuss the conditions in which there are possible damped and undamped waves. On the other hand, for longitudinal microrotation waves, after computing the speeds of propagation, we find the frequencies for which we have undamped longitudinal microrotation waves. Our results are in concordance with the results obtained in the case of purely micropolar materials $[13,14]$.

\section{PRELIMINARIES}

We consider a body which at the time $t=0$ occupies a bounded open set $B$ of the three-dimensional Euclidian space $E^{3}$ and assume that its boundary $\partial B$ is a piecewise smooth surface. A binary mixture of micropolar homogeneous thermoelastic solids fills $B$. In the following the configuration of the body at the initial time $t=0$ is considered as the reference configuration.

We refer the motion of the body to a rectangular axes $O x_{i}$. Throughout this paper, Latin subscripts take the values $1,2,3$, summation is carried out over repeated indices. Typical conventions for differential operations are implied such as a superposed dot or comma followed by a subscript to denote the partial derivative with respect to the time or to the corresponding cartesian coordinate. The superscripts $\sigma=1,2$ mark that we consider one of the micropolar elastic constituents.

In the equations of the theory of micropolar solid-solid mixtures, we use the following notations:

- $\rho^{(1)}$ and $\rho^{(2)}$ are the densities at the initial time;

- $\mathbf{u}^{(1)}$ and $\mathbf{u}^{(2)}$ are the displacement vectors;

- $\varphi^{(1)}$ and $\varphi^{(2)}$ are the microrotation vectors;

- $T$ is the variation of temperature from the reference configuration;

- $I_{i j}^{(1)}, I_{i j}^{(2)}$ are the coefficients of inertia at time $t=0$;

- $t_{i j}^{(1)}$ and $t_{i j}^{(2)}$ are the partial stress tensors;

- $m_{i j}^{(1)}$ and $m_{i j}^{(2)}$ are the partial couple stress tensors;

- $\mathbf{p}$ is the internal body force;

- $\mathbf{R}$ is the internal body couple;

- $\mathbf{F}^{(1)}$ and $\mathbf{F}^{(2)}$ are the body forces;

- $\mathbf{G}^{(1)}$ and $\mathbf{G}^{(2)}$ are the body couples;

- $T_{0}$ is the constant absolute temperature of the mixture in the reference configuration;

- $\mathbf{q}$ is the heat flux vector;

- $\eta$ is the entropy;

- $r$ is the heat supply.

As discussed in [9] (see also [8, 10]), the fundamental system of field equations that governs the motion of an anisotropic mixture consists of:

- the equations of motion

$$
\begin{aligned}
& t_{j i, j}^{(1)}-p_{i}+\rho^{(1)} F_{i}^{(1)}=\rho^{(1)} \ddot{u}_{i}^{(1)} \\
& t_{j i, j}^{(2)}+p_{i}+\rho^{(2)} F_{i}^{(2)}=\rho^{(2)} \ddot{u}_{i}^{(2)} \\
& m_{j i, j}^{(1)}+\varepsilon_{i j k}\left[t_{j k}^{(1)}+t_{j k}^{(2)}\right]-R_{i}+\rho^{(1)} G_{i}^{(1)}=\rho^{(1)} I_{i j}^{(1)} \ddot{\varphi}_{j}^{(1)} \\
& m_{j i, j}^{(2)}+R_{i}+\rho^{(2)} G_{i}^{(2)}=\rho^{(2)} I_{i j}^{(2)} \ddot{\varphi}_{j}^{(2)}
\end{aligned}
$$


in $B \times(0, \infty)$;

- the energy equation

$$
\rho T_{0} \dot{\eta}=q_{i, i}+\rho r
$$

in $B \times(0, \infty)$, where $\rho=\rho^{(1)}+\rho^{(2)}$

- the constitutive equations

$$
\begin{aligned}
t_{i j}^{(1)}= & A_{i j r s} e_{r s}+B_{i j r s} g_{r s}+F_{i j r s}^{(1)} \gamma_{r s}^{(1)}+F_{i j r s}^{(2)} \gamma_{r s}^{(2)} \\
& +a_{i j k} d_{k}+b_{i j k} \pi_{k}-\alpha_{i j}^{*} T \\
t_{i j}^{(2)}= & B_{r s i j} e_{r s}+C_{i j r s} g_{r s}+H_{i j r s}^{(1)} \gamma_{r s}^{(1)}+H_{i j r s}^{(2)} \gamma_{r s}^{(2)} \\
& +c_{i j k} d_{k}+d_{i j k} \pi_{k}-\beta_{i j}^{*} T \\
m_{i j}^{(1)}= & F_{r s i j}^{(1)} e_{r s}+H_{r s i j}^{(1)} g_{r s}+D_{i j r s}^{(1)} \gamma_{r s}^{(1)}+D_{i j r s}^{(3)} \gamma_{r s}^{(2)} \\
& +\alpha_{i j k}^{(1)} d_{k}+\beta_{i j k}^{(1)} \pi_{k}-\nu_{i j}^{*} T \\
m_{i j}^{(2)}= & F_{r s i j}^{(2)} e_{r s}+H_{r s i j}^{(2)} g_{r s}+D_{r s i j}^{(3)} \gamma_{r s}^{(1)}+D_{i j r s}^{(2)} \gamma_{r s}^{(2)} \\
& +\alpha_{i j k}^{(2)} d_{k}+\beta_{i j k}^{(2)} \pi_{k}-\mu_{i j}^{*} T \\
p_{i}= & a_{j k i} e_{j k}+c_{j k i} g_{j k}+\alpha_{j k i}^{(1)} \gamma_{j k}^{(1)}+\alpha_{j k i}^{(2)} \gamma_{j k}^{(2)} \\
& +a_{i j} d_{j}+c_{i j} \pi_{j}-\tau_{i}^{*} T \\
R_{i}= & b_{j k i} e_{j k}+d_{j k i} g_{j k}+\beta_{j k i}^{(1)} \gamma_{j k}^{(1)}+\beta_{j k i}^{(2)} \gamma_{j k}^{(2)} \\
& +c_{j i} d_{j}+b_{i j} \pi_{j}-\sigma_{i}^{*} T \\
\rho_{0} \eta= & \alpha_{i j}^{*} e_{i j}+\beta_{i j}^{*} g_{i j}+\nu_{i j}^{*} \gamma_{i j}^{(1)}+\mu_{i j}^{*} \gamma_{i j}^{(2)} \\
& +\tau_{i}^{*} d_{i}+\sigma_{i}^{*} \pi_{i}+a^{*} T \\
q_{i}= & k_{i j} T_{, j}
\end{aligned}
$$

in $\bar{B} \times[0, \infty)$, where $e_{i j}, g_{i j}, \gamma_{i j}^{(\sigma)}, d_{i}$ and $\pi_{i}$ are defined by

$$
\begin{aligned}
& e_{j i}=u_{i, j}^{(1)}+\epsilon_{s i j} \varphi_{s}^{(1)}, \quad g_{j i}=u_{i, j}^{(2)}+\epsilon_{s i j} \varphi_{s}^{(1)} \\
& d_{i}=u_{i}^{(1)}-u_{i}^{(2)}, \quad \gamma_{i j}^{(\sigma)}=\varphi_{j, i}^{(\sigma)}, \quad \pi_{i}=\varphi_{i}^{(1)}-\varphi_{i}^{(2)}
\end{aligned}
$$

The constitutive coefficients have the following symmetries

$$
\begin{aligned}
& A_{i j r s}=A_{r s i j}, \quad C_{i j r s}=C_{r s i j} \\
& D_{i j r s}^{(\sigma)}=D_{r s i j}^{(\sigma)}, \quad a_{i j}=a_{j i}, \quad b_{i j}=b_{j i}
\end{aligned}
$$

and moreover, the dissipation inequality implies

$$
\Phi \equiv \frac{1}{T_{0}} k_{i j} T_{, i} T_{, j} \geq 0
$$

To the above equations we have to adjoin boundary conditions and initial conditions. We consider null boundary conditions, i.e.

$$
u_{i}^{(\sigma)}=0, \quad \varphi_{i}^{(\sigma)}=0, \quad T=0 \quad \text { on } \partial B \times[0, \infty)
$$

We denote by $(\mathcal{P})$ the initial boundary value problem defined by Eqs $(2.1)-(2.4)$, the boundary conditions 
(2.7) and the initial conditions

$$
\begin{array}{lc}
u_{i}^{(\sigma)}(\mathbf{x}, 0)=u_{i}^{0(\sigma)}(\mathbf{x}), & \dot{u}_{i}^{(\sigma)}(\mathbf{x}, 0)=v_{i}^{0(\sigma)}(\mathbf{x}) \\
\dot{\varphi}_{i}^{(\sigma)}(\mathbf{x}, 0)=\varphi_{i}^{0(\sigma)}(\mathbf{x}), & \dot{\varphi}_{i}^{(\sigma)}(\mathbf{x}, 0)=\nu_{i}^{0(\sigma)}(\mathbf{x}) \\
T(\mathbf{x}, 0)=T^{0}(\mathbf{x}), & \mathbf{x} \in \bar{B}
\end{array}
$$

where $u_{i}^{0(\sigma)}, v_{i}^{0(\sigma)}, \varphi_{i}^{0(\sigma)}, \nu_{i}^{0(\sigma)}$, and $T^{0}$ are prescribed functions.

Let us denote by $W$ the internal energy density associated to the solution of the problem $(\mathcal{P})$, that is

$$
\begin{aligned}
W & =\frac{1}{2} A_{i j r s} e_{i j} e_{r s}+B_{i j r s} e_{i j} g_{r s}+\frac{1}{2} C_{i j r s} g_{i j} g_{r s} \\
+ & \sum_{\sigma=1}^{2}\left(\frac{1}{2} D_{i j r s}^{(\sigma)} \gamma_{i j}^{(\sigma)} \gamma_{r s}^{(\sigma)}+F_{i j r s}^{(\sigma)} e_{i j} \gamma_{r s}^{(\sigma)}+H_{i j r s}^{(\sigma)} g_{i j} \gamma_{r s}^{(\sigma)}\right) \\
& +D_{i j r s}^{(3)} \gamma_{i j}^{(1)} \gamma_{r s}^{(2)}+\frac{1}{2} a_{i j} d_{i} d_{j}+\frac{1}{2} b_{i j} \pi_{i} \pi_{j} \\
& +c_{i j} d_{i} \pi_{j}+a_{i j k} e_{i j} d_{k}+b_{i j k} e_{i j} \pi_{k} \\
& +c_{i j k} g_{i j} d_{k}+d_{i j k} g_{i j} \pi_{k}+\alpha_{i j k}^{(1)} \gamma_{i j}^{(1)} d_{k} \\
& +\beta_{i j k}^{(1)} \gamma_{i j}^{(1)} \pi_{k}+\alpha_{i j k}^{(2)} \gamma_{i j}^{(2)} d_{k}+\beta_{i j k}^{(2)} \gamma_{i j}^{(2)} \pi_{k}
\end{aligned}
$$

We assume that the internal energy density is a positive definite form and also that there exist a positive constant $k$ such that

$$
\int_{B} k_{i j} T_{, i} T_{, j} d v \geq k \int_{B} T_{, i} T_{, i} d v
$$

for all $T \in C_{0}^{\infty}(B)$.

Further, we assume that $\rho^{(\sigma)}$ and $a^{*}$ are strictly positive and the tensors $I_{i j}^{(\sigma)}$ are positive definite.

As in $[15,16]$ some new notations which involve the unknown functions and the constitutive coefficients can be introduced in order to obtain a dimensionless form of the equations. So, without losing the generality, we consider that all quantities involved in this article are dimensionless.

\section{EQUILIBRIUM THEORY}

In the absence of time dependence, the basic equations reduce to the constitutive equations (2.3), the geometrical equations (2.4) and the equations of equilibrium

$$
\begin{aligned}
& t_{j i, j}^{(1)}-p_{i}+\rho^{(1)} F_{i}^{(1)}=0 \\
& t_{j i, j}^{(2)}+p_{i}+\rho^{(2)} F_{i}^{(2)}=0 \\
& m_{j i, j}^{(1)}+\varepsilon_{i j k}\left[t_{j k}^{(1)}+t_{j k}^{(2)}\right]-R_{i}+\rho^{(1)} G_{i}^{(1)}=0 \\
& m_{j i, j}^{(2)}+R_{i}+\rho^{(2)} G_{i}^{(2)}=0 \\
& q_{i, i}=-\rho r
\end{aligned}
$$

in $B$.

The boundary conditions are

$$
u_{i}^{(\sigma)}=0, \quad \varphi_{i}^{(\sigma)}=0, \quad T=0 \quad \text { on } \partial B
$$

As in the classical thermoelasticity, the energy equation involve only the unknown temperature. Thus, in view of the assumption (2.10), we can say that for each $r \in H^{-1}(B)$ the problem defined by the equation $(3.1)_{5}$ and the boundary condition $(3.2)_{3}$ has a unique weak solution $T \in H_{0}^{1}(B)$. We recall that $H^{-1}(B)$ is the dual space of the Sobolev space $H_{0}^{1}(B)[17,18]$. 
In the following we suppose that the body loads satisfy the following regularity conditions

$$
F_{i}^{(\sigma)}, G_{i}^{(\sigma)}, r \in H^{-1}(B)
$$

In view of the above remarks, the equations of equilibrium can be rewritten in the form

$$
\begin{aligned}
& t_{j i, j}^{(1)}-p_{i}+f_{i}^{(1)}=0 \\
& t_{j i, j}^{(2)}+p_{i}+f_{i}^{(2)}=0 \\
& m_{j i, j}^{(1)}+\varepsilon_{i j k}\left[t_{j k}^{(1)}+t_{j k}^{(2)}\right]-R_{i}+g_{i}^{(1)}=0 \\
& m_{j i, j}^{(2)}+R_{i}+g_{i}^{(2)}=0
\end{aligned}
$$

where

$$
\begin{aligned}
f_{i}^{(1)} & =\rho^{(1)} F_{i}^{(1)}-\alpha_{j i}^{*} T_{, j}+\tau_{i}^{*} T \\
f_{i}^{(2)} & =\rho^{(2)} F_{i}^{(2)}-\beta_{j i}^{*} T_{, j}-\tau_{i}^{*} T \\
f_{i}^{(1)} & =\rho^{(1)} G_{i}^{(1)}-\nu_{j i}^{*} T_{, j}+\sigma_{i}^{*} T-\varepsilon_{i j k}\left(\alpha_{j k}^{*}+\beta_{j k}^{*}\right) T \\
g_{i}^{(2)} & =\rho^{(2)} G_{i}^{(2)}-\mu_{j i}^{*} T_{, j}-\sigma_{i}^{*} T
\end{aligned}
$$

Since $H_{0}^{1}(B) \subset L^{2}(B) \subset H^{-1}(B)$, it follows that

$$
f_{i}^{(\sigma)}, g_{i}^{(\sigma)} \in H^{-1}(B)
$$

Let introduce the space

$$
\mathcal{X}=\left\{\mathbf{U}=\left(u_{i}^{(1)}, \varphi_{i}^{(1)}, u_{i}^{(2)}, \varphi_{i}^{(2)}\right) \mid u_{i}^{(\sigma)}, \varphi_{i}^{(\sigma)} \in H_{0}^{1}(B)\right\}
$$

It is easy to see that

$$
\begin{aligned}
& e_{i j}(\mathbf{U}) e_{i j}(\mathbf{U}) \leq 2 u_{i, j}^{(1)} u_{i, j}^{(1)}+3 \varphi_{k}^{(1)} \varphi_{k}^{(1)} \\
& g_{i j}(\mathbf{U}) g_{i j}(\mathbf{U}) \leq 2 u_{i, j}^{(2)} u_{i, j}^{(2)}+3 \varphi_{k}^{(1)} \varphi_{k}^{(1)}
\end{aligned}
$$

where by $e_{i j}(\mathbf{U})$ we mark that $e_{i j}$ is related to the components of $\mathbf{U}$.

Moreover, using the boundary conditions, as in [19], we deduce that

$$
\int_{B} e_{i j}(\mathbf{U}) e_{i j}(\mathbf{U}) d v \geq \frac{1}{2} \int_{B}\left(u_{i, j}^{(1)} u_{i, j}^{(1)}+u_{i, j}^{(1)} u_{j, i}^{(1)}\right) d v \geq \frac{1}{2} \int_{B}\left(u_{i, j}^{(1)} u_{i, j}^{(1)}+u_{j, j}^{(1)} u_{i, i}^{(1)}\right) d v
$$

Similarly, we have

$$
\int_{B} g_{i j}(\mathbf{U}) g_{i j}(\mathbf{U}) d v \geq \frac{1}{2} \int_{B}\left(u_{i, j}^{(2)} u_{i, j}^{(2)}+u_{j, j}^{(2)} u_{i, i}^{(2)}\right) d v
$$

In view of the above relations and of the Poincaré inequality, we define on the space $\mathcal{X}$ the scalar product

$$
\langle\mathbf{U}, \mathbf{V}\rangle_{\mathcal{X}}=\int_{B}\left[e_{i j}(\mathbf{U}) e_{i j}(\mathbf{V})+g_{i j}(\mathbf{U}) g_{i j}(\mathbf{V})+\sum_{\sigma=1,2} \gamma_{i j}^{(\sigma)}(\mathbf{U}) \gamma_{i j}^{(\sigma)}(\mathbf{V})\right] d v
$$

A direct consequence of the above inequalities is the fact that the norm induced by this scalar product is equivalent with the usual norm on $\mathbf{H}_{0}^{1}(B) \times \mathbf{H}_{0}^{1}(B)$. Hence $\langle,\rangle_{\mathcal{X}}$ defines a norm equivalent to the usual norm on $\mathcal{X}$. 
We introduce the bilinear form $a: \mathcal{X} \times \mathcal{X} \rightarrow \mathbb{R}$

$$
\begin{aligned}
& a(\mathbf{U}, \mathbf{V})=A_{i j r s} e_{i j}(\mathbf{U}) e_{r s}(\mathbf{V})+C_{i j r s} g_{i j}(\mathbf{U}) g_{r s}(\mathbf{V}) \\
& \quad+B_{i j r s}\left[e_{i j}(\mathbf{U}) g_{r s}(\mathbf{V})+e_{i j}(\mathbf{V}) g_{r s}(\mathbf{U})\right] \\
& \quad+\sum_{\sigma=1}^{2}\left(D_{i j r s}^{(\sigma)} \gamma_{i j}^{(\sigma)}(\mathbf{U}) \gamma_{r s}^{(\sigma)}(\mathbf{V})+F_{i j r s}^{(\sigma)}\left[e_{i j}(\mathbf{U}) \gamma_{r s}^{(\sigma)}(\mathbf{V})+e_{i j}(\mathbf{V}) \gamma_{r s}^{(\sigma)}(\mathbf{U})\right]\right. \\
& \quad+H_{i j r s}^{(\sigma)}\left[g_{i j}(\mathbf{U}) \gamma_{r s}^{(\sigma)}(\mathbf{V})+g_{i j}(\mathbf{V}) \gamma_{r s}^{(\sigma)}(\mathbf{U})\right) \\
& \quad+D_{i j r s}^{(3)}\left[\gamma_{i j}^{(1)}(\mathbf{U}) \gamma_{r s}^{(2)}(\mathbf{V})+\gamma_{i j}^{(1)}(\mathbf{V}) \gamma_{r s}^{(2)}(\mathbf{U})\right]+a_{i j} d_{i}(\mathbf{U}) d_{j}(\mathbf{V})+b_{i j} \pi_{i}(\mathbf{U}) \pi_{j}(\mathbf{V}) \\
& \quad+c_{i j}\left[d_{i}(\mathbf{U}) \pi_{j}(\mathbf{V})+d_{i}(\mathbf{V}) \pi_{j}(\mathbf{U})\right]+a_{i j k}\left[e_{i j}(\mathbf{U}) d_{k}(\mathbf{V})+e_{i j}(\mathbf{V}) d_{k}(\mathbf{U})\right] \\
& \quad+b_{i j k}\left[e_{i j}(\mathbf{U}) \pi_{k}(\mathbf{V})+e_{i j}(\mathbf{V}) \pi_{k}(\mathbf{U})\right]+c_{i j k}\left[g_{i j}(\mathbf{U}) d_{k}(\mathbf{V})+g_{i j}(\mathbf{V}) d_{k}(\mathbf{U})\right] \\
& \quad+d_{i j k}\left[g_{i j}(\mathbf{U}) \pi_{k}(\mathbf{V})+g_{i j}(\mathbf{V}) \pi_{k}(\mathbf{U})\right]+\alpha_{i j k}^{(1)}\left[\gamma_{i j}^{(1)}(\mathbf{U}) d_{k}(\mathbf{V})+\gamma_{i j}^{(1)}(\mathbf{V}) d_{k}(\mathbf{U})\right] \\
& \quad+\beta_{i j k}^{(1)}\left[\gamma_{i j}^{(1)}(\mathbf{U}) \pi_{k}(\mathbf{V})+\gamma_{i j}^{(1)}(\mathbf{V}) \pi_{k}(\mathbf{U})\right]+\alpha_{i j k}^{(2)}\left[\gamma_{i j}^{(2)}(\mathbf{U}) d_{k}(\mathbf{V})+\gamma_{i j}^{(2)}(\mathbf{V}) d_{k}(\mathbf{U})\right] \\
& \quad+\beta_{i j k}^{(2)}\left[\gamma_{i j}^{(2)}(\mathbf{U}) \pi_{k}(\mathbf{V})+\gamma_{i j}^{(2)}(\mathbf{V}) \pi_{k}(\mathbf{U})\right]
\end{aligned}
$$

and the linear operator $F: \mathcal{X} \rightarrow \mathbb{R}$ defined by

$$
F(\mathbf{V})=\sum_{\sigma=1,2} \int_{B}\left(f_{i}^{(\sigma)} v_{i}^{(\sigma)}+g_{i}^{(\sigma)} \phi_{i}^{(\sigma)}\right) d v
$$

where $\mathbf{U}=\left(u_{i}^{(1)}, \varphi_{i}^{(1)}, u_{i}^{(2)}, \varphi_{i}^{(2)}\right) \in \mathcal{X}$ and $\mathbf{V}=\left(v_{i}^{(1)}, \phi_{i}^{(1)}, v_{i}^{(2)}, \phi_{i}^{(2)}\right) \in \mathcal{X}$.

Then the weak formulation of the problem defined by the equations (3.4) and the boundary conditions (3.2) reads as follows. Find $\mathbf{U} \in \mathcal{X}$ such that

$$
A(\mathbf{U}, \mathbf{V})=F(\mathbf{V}), \text { for all } \mathbf{V} \in \mathcal{X}
$$

where

$$
A(\mathbf{U}, \mathbf{V})=\int_{B} a(\mathbf{U}, \mathbf{V}) d v
$$

Theorem 3.1 Assume that

i) the internal density energy is a positive definite form, in term of the quantities given by (2.4);

ii) the constitutive coefficients satisfy the symmetry relations (2.5);

iii) the loads satisfy the regularity conditions (3.3).

Then there exists one and only one solution of the problem (3.14).

Proof. First of all, let remark that the hypotheses i) and ii) assure that there exist the positive constants $c_{1}$ and $c_{2}$ such that

$$
\begin{aligned}
& c_{1}\left(e_{i j}(\mathbf{U}) e_{i j}(\mathbf{U})+g_{i j}(\mathbf{U}) g_{i j}(\mathbf{U})+\sum_{\sigma=1,2} \gamma_{i j}^{(\sigma)}(\mathbf{U}) \gamma_{i j}^{(\sigma)}(\mathbf{U})+d_{i}(\mathbf{U}) d_{i}(\mathbf{U})+\pi_{i}(\mathbf{U}) \pi_{i}(\mathbf{U})\right) \leq a(\mathbf{U}, \mathbf{U}) \\
& a(\mathbf{U}, \mathbf{U}) \leq c_{2}\left(e_{i j}(\mathbf{U}) e_{i j}(\mathbf{U})+g_{i j}(\mathbf{U}) g_{i j}(\mathbf{U})+\sum_{\sigma=1,2} \gamma_{i j}^{(\sigma)}(\mathbf{U}) \gamma_{i j}^{(\sigma)}(\mathbf{U})+d_{i}(\mathbf{U}) d_{i}(\mathbf{U})+\pi_{i}(\mathbf{U}) \pi_{i}(\mathbf{U})\right)
\end{aligned}
$$

We also note that

$$
d_{i}(\mathbf{U}) d_{i}(\mathbf{U}) \leq 2 \sum_{\sigma=1,2} u_{i}^{(\sigma)} u_{i}^{(\sigma)}, \quad \pi_{i}(\mathbf{U}) \pi_{i}(\mathbf{U}) \leq 2 \sum_{\sigma=1,2} \varphi_{i}^{(\sigma)} \varphi_{i}^{(\sigma)}
$$

So, in view of the Schwarz inequality, the Poincaré inequality and the above relations, it follows that 
$A(\cdot, \cdot)$ is bounded, i.e. there exists the constant $c_{3}>0$ such that

$$
A(\mathbf{U}, \mathbf{V}) \leq c_{3}\|\mathbf{U}\|_{\mathcal{X}}\|\mathbf{V}\|_{\mathcal{X}}, \text { for all } \mathbf{U}, \mathbf{V} \in \mathcal{X}
$$

Moreover, $(3.16)_{1}$ implies that $A(\cdot, \cdot)$ is coercive, i.e there exists the positive constant $c_{4}$ such that

$$
A(\mathbf{U}, \mathbf{U}) \geq c_{4}\|\mathbf{U}\|_{\mathcal{X}}^{2}, \quad \text { for all } \mathbf{U} \in \mathcal{X}
$$

Finally, because the injections of $L^{2}(B)$ into $H^{-1}(B)$ are continuous, the Schwarz inequality imply that the linear operator $F(\cdot)$ is bounded. By the LaxMilgram theorem it follows that (3.14) has one and only one solution. The proof is complete.

Remark 3.1 The Lax-Milgram theorem used in the proof of the previous theorem also offers a continuous dependence result on the loads $f_{i}^{(\sigma)}, g_{i}^{(\sigma)}$. The map $\left(f_{i}^{(\sigma)}, g_{i}^{(\sigma)}\right) \rightarrow\left(u_{i}^{(\sigma)}, \varphi_{i}^{(\sigma)}\right)$ is Lipschitzian from $\left[H^{-1}(B)\right]^{6}$ to $\mathcal{X}$ with the Lipschitz constant $c_{4}^{-1}$. Moreover, the weak solution $\mathbf{U}$ minimizes the functional $\frac{1}{2} A(\mathbf{U}, \mathbf{U})-F(\mathbf{U})$ on $\mathcal{X}$.

\section{DYNAMIC THEORY}

In this section we use the results of the semigroup theory of linear operators to establish an existence theorem in the dynamical theory of mixtures of two micropolar elastic solids. First, we transform our initial boundary value problem defined by the relations $(2.1)-(2.4),(2.7)$ and $(2.8)$ to an abstract problem on an appropriate Hilbert space.

Let us define

$$
\mathcal{Z}=\left\{\mathbf{W}=\left(u_{i}^{(1)}, v_{i}^{(1)}, \varphi_{i}^{(1)}, \nu_{i}^{(1)}, u_{i}^{(2)}, v_{i}^{(2)}, \varphi_{i}^{(2)}, \nu_{i}^{(2)}, T\right) \mid u_{i}^{(\sigma)}, \varphi_{i}^{(\sigma)} \in H_{0}^{1}(B), \quad v_{i}^{(\sigma)}, \nu_{i}^{(\sigma)}, T \in L^{2}(B)\right\}
$$

We consider the operators

$$
\begin{gathered}
A_{i}^{(1)}\left(\mathbf{u}^{(1)}\right)=\frac{1}{\rho^{(1)}}\left[\left(A_{j i r s} u_{s, r}^{(1)}+a_{j i k} u_{k}^{(1)}\right)_{, j}-a_{i j} u_{j}^{(1)}-a_{j k i} u_{k, j}^{(1)}\right] \\
A_{i}^{(2)}\left(\mathbf{u}^{(2)}\right)=\frac{1}{\rho^{(1)}}\left[\left(B_{j i r s} u_{s, r}^{(2)}-a_{j i k} u_{k}^{(2)}\right)_{, j}+a_{i j} u_{j}^{(2)}-c_{j k i} u_{k, j}^{(2)}\right] \\
B_{i}^{(1)}\left(\varphi^{(1)}\right)=\frac{1}{\rho^{(1)}}\left[\left(\left(A_{j i r s}+B_{j i r s}\right) \varepsilon_{m s r} \varphi_{m}^{(1)}+F_{j i r s}^{(1)} \varphi_{s, r}^{(1)}+b_{j i k} \varphi_{k}^{(1)}\right)_{, j}\right. \\
\left.-c_{i j} \varphi_{j}^{(1)}-\left(a_{j k i}+c_{j k i}\right) \varepsilon_{m k j} \varphi_{m}^{(1)}-\alpha_{j k i}^{(1)} \varphi_{k, j}^{(1)}\right] \\
B_{i}^{(2)}\left(\varphi^{(2)}\right)=\frac{1}{\rho^{(1)}}\left[\left(F_{j i r s}^{(2)} \varphi_{s, r}^{(2)}-b_{j i k} \varphi_{k}^{(2)}\right)_{, j}-c_{i j} \varphi_{j}^{(2)}-\alpha_{j k i}^{(2)} \varphi_{k, j}^{(2)}\right] \\
D_{i}^{(1)}\left(\mathbf{u}^{(1)}\right)=\frac{J_{i m}^{(1)}\left[()^{(1)}\right.}{\rho^{(1)}} \frac{1}{\rho^{(1)}}\left[\left(-\alpha_{j i}^{*} T\right)_{, j}+\tau_{i}^{*} T\right] \\
\left.-c_{j m}^{(1)} u_{j}^{(1)}-b_{j k m} u_{s, r}^{(1)}+\alpha_{j m k}^{(1)} u_{k}^{(1)}\right)_{, j}+\varepsilon_{j m k}\left[\left(A_{j k r s}+B_{r s j k}\right) u_{s, r}^{(1)}+\left(a_{j k n}+c_{j k n}\right) u_{n}^{(1)}\right] \\
D_{i}^{(2)}\left(\mathbf{u}^{(2)}\right)=\frac{J_{i m}^{(1)}}{\rho^{(1)}}\left[\left(H_{r s j m}^{(1)} u_{s, r}^{(2)}-\alpha_{j m k}^{(1)} u_{k}^{(2)}\right)_{, j}+\varepsilon_{j m k}\left[\left(B_{j k r s}+C_{j k r s}\right) u_{s, r}^{(2)}-\left(a_{j k n}+c_{j k n}\right) u_{n}^{(2)}\right]\right. \\
\left.-c_{j m} u_{j}^{(2)}-d_{j k m} u_{k, j}^{(2)}\right]
\end{gathered}
$$




$$
\begin{aligned}
& E_{i}^{(1)}\left(\varphi^{(1)}\right)=\frac{J_{i p}^{(1)}}{\rho^{(1)}}\left[\left[\left(F_{r s j p}^{(1)}+H_{r s j p}^{(1)}\right) \varepsilon_{m s r} \varphi_{m}^{(1)}+D_{r s j p}^{(1)} \varphi_{s, r}^{(1)}+\beta_{j p k}^{(1)} \varphi_{k}^{(1)}\right)_{, j}\right. \\
& +\varepsilon_{p j k}\left[\left(A_{j k r s}+B_{j k r s}+B_{r s j k}+C_{j k r s}\right) \varepsilon_{m s r} \varphi_{m}^{(1)}+\left(b_{j k m}+d_{j k m}\right) \varphi_{m}^{(1)}+\left(F_{j k r s}+H_{j k r s}^{(1)}\right) \varphi_{s, r}^{(1)}\right] \\
& \left.-b_{p j} \varphi_{j}^{(1)}-\left(b_{j k p}+d_{j k p}\right) \varepsilon_{k j m} \varphi_{m}^{(1)}-\beta_{j k p}^{(1)} \varphi_{k, j}^{(1)}\right] \\
& E_{i}^{(2)}\left(\boldsymbol{\varphi}^{(2)}\right)=\frac{J_{i p}^{(1)}}{\rho^{(1)}}\left[\left(D_{j p r s}^{(3)} \varphi_{s, r}^{(2)}-\beta_{j p k}^{(1)} \varphi_{k}^{(2)}\right)_{, j}+\varepsilon_{p j k}\left[\left(F_{j k r s}^{(2)}+H_{j k r s}^{(2)}\right) \varphi_{s, r}^{(2)}-\left(b_{j k m}+d_{j k m}\right) \varphi_{m}^{(2)}\right]\right. \\
& \left.-b_{p j} \varphi_{j}^{(2)}-\beta_{j k p}^{(2)} \varphi_{k, j}^{(2)}\right] \\
& F_{i}(T)=\frac{J_{i m}^{(1)}}{\rho^{(1)}}\left[\left(-\nu_{j m}^{*} T\right)_{, j}-\varepsilon_{m j k}\left(\alpha_{j k}^{*}+\beta_{j k}^{*}\right) T+\sigma_{m}^{*} T\right] \\
& G_{i}^{(1)}\left(\mathbf{u}^{(1)}\right)=\frac{1}{\rho^{(2)}}\left[\left(B_{r s j i} u_{s, r}^{(1)}+c_{j i k} u_{k}^{(1)}\right)_{, j}+a_{i j} u_{j}^{(1)}+a_{j k i} u_{k, j}^{(1)}\right] \\
& G_{i}^{(2)}\left(\mathbf{u}^{(2)}\right)=\frac{1}{\rho^{(2)}}\left[\left(C_{j i r s} u_{s, r}^{(2)}-c_{j i k} u_{k}^{(2)}\right)_{, j}-a_{i j} u_{j}^{(2)}+c_{j k i} u_{k, j}^{(2)}\right] \\
& H_{i}^{(1)}\left(\varphi^{(1)}\right)=\frac{1}{\rho^{(2)}}\left[\left(\left(B_{r s j i}+C_{r s j i}\right) \varepsilon_{m s r} \varphi_{m}^{(1)}+H_{j i r s}^{(1)} \varphi_{s, r}^{(1)}+d_{j i k} \varphi_{k}^{(1)}\right)_{, j}\right. \\
& \left.+c_{i j} \varphi_{j}^{(1)}+\left(a_{j k i}+c_{j k i}\right) \varepsilon_{m k j} \varphi_{m}^{(1)}+\alpha_{j k i}^{(1)} \varphi_{k, j}^{(1)}\right] \\
& H_{i}^{(2)}\left(\boldsymbol{\varphi}^{(1)}\right)=\frac{1}{\rho^{(2)}}\left[\left(H_{j i r s}^{(2)} \varphi_{s, r}^{(2)}-d_{j i k} \varphi_{k}^{(2)}\right)_{, j}+\alpha_{j k i}^{(2)} \varphi_{k, j}^{(2)}-c_{i j} \varphi_{j}^{(2)}\right] \\
& K_{i}(T)=\frac{1}{\rho^{(2)}}\left[\left(-\beta_{j i}^{*} T\right)_{, j}-\tau_{i}^{*} T\right] \\
& Q_{i}^{(1)}\left(\mathbf{u}^{(1)}\right)=\frac{J_{i m}^{(2)}}{\rho^{(2)}}\left[\left(F_{r s j m}^{(2)} u_{s, r}^{(1)}+\alpha_{j m k}^{(2)} u_{k}^{(1)}\right)_{, j}+c_{j m} u_{j}^{(1)}+b_{j k m} u_{k, j}^{(1)}\right] \\
& Q_{i}^{(2)}\left(\mathbf{u}^{(2)}\right)=\frac{J_{i m}^{(2)}}{\rho^{(2)}}\left[\left(H_{r s j m}^{(2)} u_{s, r}^{(2)}-\alpha_{j m k}^{(2)} u_{k}^{(2)}\right)_{, j}-c_{j m} u_{j}^{(2)}+d_{j k m} u_{k, j}^{(2)}\right] \\
& P_{i}^{(1)}\left(\varphi^{(1)}\right)=\frac{J_{i p}^{(2)}}{\rho^{(2)}}\left[\left[\left(F_{r s j p}^{(2)}+H_{r s j p}^{(2)}\right) \varepsilon_{m s r} \varphi_{m}^{(1)}+D_{r s j p}^{(3)} \varphi_{s, r}^{(1)}+\beta_{j p k}^{(2)} \varphi_{k}^{(1)}\right)_{, j}\right. \\
& \left.+b_{p j} \varphi_{j}^{(2)}+\left(b_{j k p}+d_{j k p}\right) \varepsilon_{k j m} \varphi_{m}^{(1)}+\beta_{j k p}^{(1)} \varphi_{k, j}^{(1)}\right] \\
& P_{i}^{(2)}\left(\boldsymbol{\varphi}^{(2)}\right)=\frac{J_{i p}^{(2)}}{\rho^{(2)}}\left[\left(D_{j p r s}^{(2)} \varphi_{s, r}^{(2)}-\beta_{j p k} \varphi_{k}^{(2)}\right)_{, j}-b_{p j} \varphi_{j}^{(2)}+\beta_{j k p} \varphi_{k, j}^{(2)}\right] \\
& F_{i}(T)=\frac{J_{i m}^{(2)}}{\rho^{(2)}}\left[\left(-\mu_{j m}^{*} T\right)_{, j}-\sigma_{m}^{*} T\right] \\
& M^{(1)}\left(\mathbf{u}^{(1)}\right)=-\frac{1}{a^{*}}\left[\alpha_{i j}^{*} v_{j, i}^{(1)}+\tau_{i}^{*} v_{i}^{(1)}\right] \\
& M^{(2)}\left(\mathbf{u}^{(2)}\right)=-\frac{1}{a^{*}}\left[\beta_{i j}^{*} v_{j, i}^{(2)}-\tau_{i}^{*} v_{i}^{(2)}\right] \\
& N^{(1)}\left(\varphi^{(1)}\right)=-\frac{1}{a^{*}}\left[\left(\alpha_{i j}^{*}+\beta_{i j}^{*}\right) \varepsilon_{m j i} \nu_{m}^{(1)}+\nu_{i j}^{*} \nu_{j, i}^{(1)}+\sigma_{i}^{*} \nu_{i}^{(1)}\right]
\end{aligned}
$$




$$
\begin{gathered}
N^{(2)}\left(\varphi^{(2)}\right)=-\frac{1}{a^{*}}\left[\mu_{i j}^{*} \nu_{j, i}^{(2)}-\sigma_{i}^{*} \nu_{i}^{(2)}\right] \\
S(T)=\frac{1}{T_{0} a^{*}}\left[k_{i j} T_{, j}\right]_{, i}
\end{gathered}
$$

where $\mathbf{J}^{(\sigma)}=\left(J_{i j}^{(\sigma)}\right)$ denotes the inverse of the matrix $\mathbf{I}^{(\sigma)}=\left(I_{i j}^{(\sigma)}\right)$ and all the derivatives of the functions are understood in the sense of distributions.

With the help of these operators, we define the matrix operator $\mathcal{A}$ with the domain $\mathcal{D}=\{\mathbf{W} \in \mathcal{Z} \mid$ $\mathcal{A} \mathbf{W} \in \mathcal{Z}\}$, by

$$
\mathcal{A}=\left(\begin{array}{ccccccccc}
0 & \mathbf{I d} & 0 & 0 & 0 & 0 & 0 & 0 & 0 \\
\mathbf{A}^{(1)} & 0 & \mathbf{B}^{(1)} & 0 & \mathbf{A}^{(2)} & 0 & \mathbf{B}^{(2)} & 0 & \mathbf{C} \\
0 & 0 & 0 & \mathbf{I d} & 0 & 0 & 0 & 0 & 0 \\
\mathbf{D}^{(1)} & 0 & \mathbf{E}^{(1)} & 0 & \mathbf{D}^{(2)} & 0 & \mathbf{E}^{(2)} & 0 & \mathbf{F} \\
0 & 0 & 0 & 0 & 0 & \mathbf{I d} & 0 & 0 & 0 \\
\mathbf{G}^{(1)} & 0 & \mathbf{H}^{(1)} & 0 & \mathbf{G}^{(2)} & 0 & \mathbf{H}^{(2)} & 0 & \mathbf{K} \\
0 & 0 & 0 & 0 & 0 & 0 & 0 & \mathbf{I d} & 0 \\
\mathbf{Q}^{(1)} & 0 & \mathbf{P}^{(1)} & 0 & \mathbf{Q}^{(2)} & 0 & \mathbf{P}^{(2)} & 0 & \mathbf{R} \\
0 & M^{(1)} & 0 & N^{(1)} & 0 & M^{(2)} & 0 & N^{(2)} & S
\end{array}\right)
$$

where $\mathbf{A}$ denote the operator $\mathbf{A}=\left(A_{1}, A_{2}, A_{3}\right)$ and $\mathbf{I d}$ is the identity operator.

The boundary initial value problem defined by the relations $(2.1)-(2.4),(2.7)$ and $(2.8)$ can be transformed into the following abstract equation in the Hilbert space $\mathcal{Z}$

$$
\frac{d \mathbf{W}}{d t}(t)=\mathcal{A} \mathbf{W}(t)+\mathcal{F}(t), \quad \mathbf{W}(0)=\mathbf{W}_{0}
$$

where

$$
\mathcal{F}(t)=\left(\mathbf{0}, \mathbf{F}^{(1)}, \mathbf{0}, \mathbf{J}^{(1)} \mathbf{G}^{(1)}, \mathbf{0}, \mathbf{F}^{(2)}, \mathbf{0}, \mathbf{J}^{(2)} \mathbf{G}^{(2)}, \frac{\rho}{T_{0} a^{*}} r\right)
$$

and

$$
\mathbf{W}_{0}=\left(\mathbf{u}^{0(1)}, \mathbf{v}^{0(1)}, \varphi^{0(1)}, \boldsymbol{\nu}^{0(1)}, \mathbf{u}^{0(2)}, \mathbf{v}^{0(2)}, \varphi^{0(2)}, \boldsymbol{\nu}^{0(2)}, T^{0}\right)
$$

We introduce the following inner product in $\mathcal{Z}$

$$
\langle\mathbf{W}, \widetilde{\mathbf{W}}\rangle_{\mathcal{Z}}=\int_{B}\left\{\sum_{\sigma=1,2}\left(\rho^{(\sigma)} v_{i}^{(\sigma)} \widetilde{v}_{i}^{(\sigma)}+\rho^{(\sigma)} I_{i j}^{(\sigma)} \nu_{i}^{(\sigma)} \widetilde{\nu}_{j}^{(\sigma)}\right)+a^{*} T \tilde{T}+a(\widehat{\mathbf{W}}, \widehat{\widehat{\mathbf{W}}})\right\} d v
$$

where $\mathbf{W}=\left(u_{i}^{(1)}, v_{i}^{(1)}, \varphi_{i}^{(1)}, \nu_{i}^{(1)}, u_{i}^{(2)}, v_{i}^{(2)}, \varphi_{i}^{(2)}, \nu_{i}^{(2)}, T\right), \widetilde{\mathbf{W}}=\left(\tilde{u}_{i}^{(1)}, \tilde{v}_{i}^{(1)}, \tilde{\varphi}_{i}^{(1)}, \tilde{\nu}_{i}^{(1)}, \tilde{u}_{i}^{(2)}, \tilde{v}_{i}^{(2)}, \tilde{\varphi}_{i}^{(2)}, \tilde{\nu}_{i}^{(2)}, \tilde{T}\right)$, the bilinear form $a(\cdot, \cdot)$ is defined by $(3.12)$ and we denote by $\widehat{\mathbf{W}}$ the vector obtained from a vector $\mathbf{W}$ by cutting the components $v_{i}^{(1)}, \nu_{i}^{(1)}, v_{i}^{(2)}, \nu_{i}^{(2)}$ and $T$.

A direct consequence of the inequalities (3.8), (3.9), (3.10), (3.16) and (3.17) is the fact that the norm induced the above inner product defines a norm equivalent to the usual norm on $\mathcal{Z}$.

We note that $\left[C_{0}^{\infty}(B)\right]^{25}$ is a dense subset of $\mathcal{Z}$ which is contained in $\mathcal{D}(\mathcal{A})$.

Lemma 4.1 The operator $\mathcal{A}$ is dissipative, i.e.

$$
\langle\mathcal{A} \mathbf{W}, \mathbf{W}\rangle_{\mathcal{Z}} \leq 0, \text { for all } \mathbf{W} \in \mathcal{D}(\mathcal{A})
$$

Proof. Using the divergence theorem and the boundary conditions we find 


$$
\begin{aligned}
\langle\mathcal{A} \mathbf{W}, \mathbf{W}\rangle_{\mathcal{Z}}= & \int_{B}\left\{v_{i}^{(1)}\left(t_{j i, j}^{(1)}-p_{i}\right)+v_{i}^{(2)}\left(t_{j i, j}^{(2)}+p_{i}\right)\right. \\
& +\nu_{i}^{(1)}\left[m_{j i, j}^{(1)}+\varepsilon_{i j k}\left(t_{j k}^{(1)}+t_{j k}^{(2)}\right)-R_{i}\right]+\nu_{i}^{(2)}\left(m_{j i, j}^{(2)}+R_{i}\right) \\
& \left.+\left[\frac{1}{T_{0}} q_{i, i}+M^{(1)}\left(\mathbf{u}^{(1)}\right)+M^{(2)}\left(\mathbf{u}^{(2)}\right)+N^{(1)}\left(\varphi^{(1)}\right)+N^{(2)}\left(\boldsymbol{\varphi}^{(2)}\right)\right] T+a(\widehat{\mathcal{A W}}, \widehat{\mathbf{W}})\right\} d v \\
= & -\int_{B} k_{i j} T_{, i} T_{, j} d v
\end{aligned}
$$

for all $\mathbf{W}=\left(u_{i}^{(1)}, v_{i}^{(1)}, \varphi_{i}^{(1)}, \nu_{i}^{(1)}, u_{i}^{(2)}, v_{i}^{(2)}, \varphi_{i}^{(2)}, \nu_{i}^{(2)}, T\right) \in \mathcal{D}(\mathcal{A})$.

Thus, using the inequality (2.10) we obtain the relation (4.31) and the proof is complete.

Lemma 4.2 The operator $\mathcal{A}$ satisfies the condition

$$
\operatorname{Rang}(\mathbf{I} \mathbf{d}-\mathcal{A})=\mathcal{Z}
$$

Proof. Let us consider $\mathbf{W}^{*}=\left(u_{i}^{*(1)}, v_{i}^{*(1)}, \varphi_{i}^{*(1)}, \nu_{i}^{*(1)}, u_{i}^{*(2)}, v_{i}^{*(2)}, \varphi_{i}^{*(2)}, \nu_{i}^{*(2)}, T^{*}\right) \in \mathcal{Z}$. We have to show that there is a solution $\mathbf{W}=\left(u_{i}^{(1)}, v_{i}^{(1)}, \varphi_{i}^{(1)}, \nu_{i}^{(1)}, u_{i}^{(2)}, v_{i}^{(2)}, \varphi_{i}^{(2)}, \nu_{i}^{(2)}, T\right) \in \mathcal{D}(\mathcal{A})$ of the equation

$$
\mathbf{W}-\mathcal{A} \mathbf{W}=\mathbf{W}^{*}
$$

From the definition of the operator $\mathcal{A}$ we get

$$
\begin{aligned}
& v_{i}^{(1)}=u_{i}^{(1)}-u_{i}^{*(1)}, \quad \nu_{i}^{(1)}=\varphi_{i}^{(1)}-\varphi_{i}^{*(1)}, \quad v_{i}^{(2)}=u_{i}^{(2)}-u_{i}^{*(2)}, \quad \nu_{i}^{(2)}=\varphi_{i}^{(2)}-\varphi_{i}^{*(2)} \\
& v_{i}^{(1)}-\left[\sum_{\sigma=1,2}\left(A_{i}^{(\sigma)}\left(\mathbf{u}^{(\sigma)}\right)+B_{i}^{(\sigma)}\left(\boldsymbol{\varphi}^{(\sigma)}\right)\right)+C_{i}(T)\right]=v_{i}^{*(1)} \\
& \nu_{i}^{(1)}-\left[\sum_{\sigma=1,2}\left(D_{i}^{(\sigma)}\left(\mathbf{u}^{(\sigma)}\right)+E_{i}^{(\sigma)}\left(\boldsymbol{\varphi}^{(\sigma)}\right)\right)+F_{i}(T)\right]=\nu_{i}^{*(1)} \\
& v_{i}^{(2)}-\left[\sum_{\sigma=1,2}\left(G_{i}^{(\sigma)}\left(\mathbf{u}^{(\sigma)}\right)+H_{i}^{(\sigma)}\left(\boldsymbol{\varphi}^{(\sigma)}\right)\right)+K_{i}(T)\right]=v_{i}^{*(2)} \\
& \nu_{i}^{(2)}-\left[\sum_{\sigma=1,2}\left(Q_{i}^{(\sigma)}\left(\mathbf{u}^{(\sigma)}\right)+P_{i}^{(\sigma)}\left(\boldsymbol{\varphi}^{(\sigma)}\right)\right)+R_{i}(T)\right]=\nu_{i}^{*(2)} \\
& T-\left[\sum_{\sigma=1,2}\left(M^{(\sigma)}\left(\mathbf{v}^{(\sigma)}\right)+N^{(\sigma)}\left(\boldsymbol{\nu}^{(\sigma)}\right)\right)+S(T)\right]=T^{*}
\end{aligned}
$$

By eliminating $v_{i}^{(1)}, \nu_{i}^{(1)}, v_{i}^{(2)}, \nu_{i}^{(2)}$, we obtain for the determination of the functions $\check{\mathbf{W}}=\left(u_{i}^{(1)}, \varphi_{i}^{(1)}, u_{i}^{(2)}, \varphi_{i}^{(2)}, T\right)$ the following system

$$
\begin{aligned}
& L_{i}^{(1)}(\check{\mathbf{W}}) \equiv u_{i}^{(1)}-\left[\sum_{\sigma=1,2}\left(A_{i}^{(\sigma)}\left(\mathbf{u}^{(\sigma)}\right)+B_{i}^{(\sigma)}\left(\boldsymbol{\varphi}^{(\sigma)}\right)\right)+C_{i}(T)\right]=g_{i}^{(1)} \\
& K_{i}^{(1)}(\check{\mathbf{W}}) \equiv \varphi_{i}^{(1)}-\left[\sum_{\sigma=1,2}\left(D_{i}^{(\sigma)}\left(\mathbf{u}^{(\sigma)}\right)+E_{i}^{(\sigma)}\left(\boldsymbol{\varphi}^{(\sigma)}\right)\right)+F_{i}(T)\right]=h_{i}^{(1)} \\
& L_{i}^{(2)}(\check{\mathbf{W}}) \equiv u_{i}^{(2)}-\left[\sum_{\sigma=1,2}\left(G_{i}^{(\sigma)}\left(\mathbf{u}^{(\sigma)}\right)+H_{i}^{(\sigma)}\left(\boldsymbol{\varphi}^{(\sigma)}\right)\right)+K_{i}(T)\right]=g_{i}^{(2)} \\
& K_{i}^{(2)}(\check{\mathbf{W}}) \equiv \varphi_{i}^{(2)}-\left[\sum_{\sigma=1,2}\left(Q_{i}^{(\sigma)}\left(\mathbf{u}^{(\sigma)}\right)+P_{i}^{(\sigma)}\left(\boldsymbol{\varphi}^{(\sigma)}\right)\right)+R_{i}(T)\right]=h_{i}^{(2)} \\
& Y(\check{\mathbf{W}}) \equiv T-\left[\sum_{\sigma=1,2}\left(M^{(\sigma)}\left(\mathbf{u}^{(\sigma)}\right)+N^{(\sigma)}\left(\boldsymbol{\varphi}^{(\sigma)}\right)\right)+S(T)\right]=s
\end{aligned}
$$


where

$$
\begin{aligned}
& g_{i}^{(\sigma)}=u_{i}^{*(\sigma)}+v_{i}^{*(\sigma)}, \quad h_{i}^{(\sigma)}=\varphi_{i}^{*(\sigma)}+\nu_{i}^{*(\sigma)} \\
& s=T^{*}-\left[\sum_{\sigma=1,2}\left(M^{(\sigma)}\left(\mathbf{u}^{*(\sigma)}\right)+N^{(\sigma)}\left(\boldsymbol{\varphi}^{*(\sigma)}\right)\right)\right]
\end{aligned}
$$

On the space $\left[H_{0}^{1}(B)\right]^{13}$, we introduce the bilinear form

$$
\begin{aligned}
\mathcal{B}(\check{\mathbf{W}}, \check{\widetilde{\mathbf{W}}})= & \left\langle\left(\mathbf{L}^{(1)}(\check{\mathbf{W}}), \mathbf{K}^{(1)}(\check{\mathbf{W}}), \mathbf{L}^{(2)}(\check{\mathbf{W}}), \mathbf{K}^{(2)}(\check{\mathbf{W}}), Y(\check{\mathbf{W}})\right),\right. \\
& \left.\left(\rho^{(1)} \widetilde{\mathbf{u}}^{(1)}, \rho^{(1)} \mathbf{I}^{(1)} \widetilde{\varphi}^{(1)}, \rho^{(1)} \widetilde{\mathbf{u}}^{(1)}, \rho^{(2)} \mathbf{I}^{(2)} \widetilde{\varphi}^{(2)}, a^{*} \widetilde{T}\right)\right\rangle_{\left[L^{2}(B)\right]^{13}}
\end{aligned}
$$

where $\widetilde{\mathbf{W}}=\left(\widetilde{u}_{i}^{(1)}, \widetilde{v}_{i}^{(1)}, \widetilde{\varphi}_{i}^{(1)}, \widetilde{\nu}_{i}^{(1)}, \widetilde{u}_{i}^{(2)}, \widetilde{v}_{i}^{(2)}, \widetilde{\varphi}_{i}^{(2)}, \widetilde{\nu}_{i}^{(2)}, \widetilde{T}\right) \in \mathcal{Z}$ and the linear operator $l:\left[H_{0}^{1}(B)\right]^{13} \rightarrow \mathbb{R}$

$$
l(\widetilde{\mathbf{y}})=\left\langle\left(\mathbf{g}^{(1)}, \mathbf{h}^{(1)}, \mathbf{g}^{(2)}, \mathbf{h}^{(2)}, s\right),\left(\rho^{(1)} \widetilde{\mathbf{u}}^{(1)}, \rho^{(1)} \mathbf{I}^{(1)} \widetilde{\boldsymbol{\varphi}}^{(1)}, \rho^{(1)} \widetilde{\mathbf{u}}^{(1)}, \rho^{(2)} \mathbf{I}^{(2)} \widetilde{\boldsymbol{\varphi}}^{(2)}, a^{*} \widetilde{T}\right)\right\rangle_{\left[L^{2}(B)\right]^{13}}
$$

In view of (3.8), (3.9), (3.10), (3.16), (3.17), the Schwarz inequality and the Poincaré inequality it is easy to see that $\mathcal{B}$ is bounded, i.e.

$$
\mathcal{B}(\check{\mathbf{W}}, \check{\widetilde{\mathbf{W}}}) \leq M_{1}\|\check{\mathbf{W}}\|_{\left[H_{0}^{1}(B)\right]^{13}}\|\check{\widetilde{\mathbf{W}}}\|_{\left[H_{0}^{1}(B)\right]^{13}}, \quad M_{1}=\text { positive constant }
$$

and coercive

$$
\begin{gathered}
\mathcal{B}(\check{\mathbf{W}}, \check{\mathbf{W}})=\int_{B}\left\{\sum_{\sigma=1,2}\left(\rho^{(\sigma)} u_{i}^{(\sigma)} u_{i}^{(\sigma)}+\rho^{(\sigma)} I_{i j}^{(\sigma)} \varphi_{i}^{(\sigma)} \varphi_{j}^{(\sigma)}\right)+a^{*} T^{2}+a(\widehat{\mathbf{W}}, \widehat{\mathbf{W}})+\frac{1}{T_{0}} k_{i j} T_{, i} T_{, j}\right\} d v \\
\geq M_{2}\|\check{\mathbf{W}}\|_{\left[H_{0}^{1}(B)\right]^{13}}^{2}, \quad M_{2}=\text { positive constant }
\end{gathered}
$$

while the linear operator $l$ is bounded

$$
l(\check{\mathbf{W}}) \leq M_{3}\|\check{\mathbf{W}}\|_{\left[H_{0}^{1}(B)\right]^{13}}, \quad M_{3}=\text { positive constant }
$$

The Lax-Milgram theorem proves the existence of the solution $\breve{\mathbf{W}} \in\left[H_{0}^{1}(B)\right]^{13}$ of the system (4.36). By taking $v_{i}^{(1)}=u_{i}^{(1)}-u_{i}^{*(1)}, \nu_{i}^{(1)}=\varphi_{i}^{(1)}-\varphi_{i}^{*(1)}, v_{i}^{(2)}=u_{i}^{(2)}-u_{i}^{*(2)}, \nu_{i}^{(2)}=\varphi_{i}^{(2)}-\varphi_{i}^{*(2)}$ we deduce that $\mathbf{W} \in\left[H_{0}^{1}(B)\right]^{25}$ is solution of the equation (4.34). The proof is complete.

Theorem 4.1 The operator $\mathcal{A}$ defined by (4.27) generates a $C_{0}$ contractive semigroup in $\mathcal{Z}$.

Proof. The proof follows using the previous lemmas and the Lumer-Phillips theorem [20].

Using the results concerning the abstract Cauchy problem [20,21], we obtain the following result:

Theorem 4.2 Assume that $F_{i}^{(\sigma)}, G_{i}^{(\sigma)}, r \in C^{1}\left(\left[0, t_{1}\right) ; L^{2}(B)\right), \mathbf{W}_{0} \in \mathcal{D}(\mathcal{A})$. Then, there exist a unique solution $\mathbf{W} \in \mathbf{C}^{1}\left(\left(0, t_{1}\right) ; \mathcal{Z}\right) \cap \mathbf{C}^{0}\left(\left[0, t_{1}\right) ; \mathcal{D}(\mathcal{A})\right)$ of the Cauchy problem $(4.28)$.

Moreover, since the semigroup generated by $\mathcal{A}$ is contractive we have the following estimate which proves the continuous dependence of the solution upon initial data an body forces.

Corollary 4.1 In the hypotheses of Theorem 4.2 we have the following estimate

$$
\|\mathbf{W}(t)\|_{\mathcal{Z}} \leq\left\|\mathbf{W}_{0}\right\|_{\mathcal{Z}}+M_{4} \int_{0}^{t}\left[\sum_{\sigma=1,2}\left(\left\|\mathbf{F}^{(\sigma)}(\tau)\right\|_{\left[L^{2}(B)\right]^{3}}+\left\|\mathbf{G}^{(\sigma)}(\tau)\right\|_{\left[L^{2}(B)\right]^{3}}\right)+\|r(\tau)\|_{L^{2}(B)}\right] d \tau
$$

where $M_{4}$ is a positive constant. 


\section{PLANE WAVES}

In this section we study plane harmonic waves in the theory of elastic isotropic mixtures when body forces and body couples are absent.

Considering plane waves propagated in the $x_{1}$-direction, we have

$$
u_{i}^{(\sigma)}=u_{i}^{(\sigma)}\left(x_{1}, t\right), \quad \varphi_{i}^{(\sigma)}=\varphi_{i}^{(\sigma)}\left(x_{1}, t\right)
$$

The field equations are $[8]$

$$
\begin{gathered}
(\lambda+2 \mu+\kappa) \frac{\partial^{2}}{\partial x_{1}^{2}} u_{1}^{(1)}+\left(\lambda_{0}+2 \mu_{0}+\kappa_{0}\right) \frac{\partial^{2}}{\partial x_{1}^{2}} u_{1}^{(2)}-\tau u_{1}^{(1)}+\tau u_{1}^{(2)}=\rho^{(1)} \frac{\partial^{2}}{\partial t^{2}} u_{1}^{(1)} \\
\left(\lambda_{0}+2 \mu_{0}+\kappa_{0}\right) \frac{\partial^{2}}{\partial x_{1}^{2}} u_{1}^{(1)}+(\bar{\lambda}+2 \bar{\mu}+\bar{\kappa}) \frac{\partial^{2}}{\partial x_{1}^{2}} u_{1}^{(2)}+\tau u_{1}^{(1)}-\tau u_{1}^{(2)}=\rho^{(2)} \frac{\partial^{2}}{\partial t^{2}} u_{1}^{(2)} \\
(\alpha+\beta+\gamma) \frac{\partial^{2}}{\partial x_{1}^{2}} \varphi_{1}^{(1)}+\left(\alpha_{0}+\beta_{0}+\gamma_{0}\right) \frac{\partial^{2}}{\partial x_{1}^{2}} \varphi_{1}^{(2)}-\alpha_{35} \varphi_{1}^{(1)}-\alpha_{36} \varphi_{1}^{(2)}=J^{(1)} \frac{\partial^{2}}{\partial t^{2}} \varphi_{1}^{(1)} \\
\left(\alpha_{0}+\beta_{0}+\gamma_{0}\right) \frac{\partial^{2}}{\partial x_{1}^{2}} \varphi_{1}^{(1)}+(\bar{\alpha}+\bar{\beta}+\bar{\gamma}) \frac{\partial^{2}}{\partial x_{1}^{2}} \varphi_{1}^{(2)}-\alpha_{43} \varphi_{1}^{(1)}-\alpha_{44} \varphi_{1}^{(2)}=J^{(2)} \frac{\partial^{2}}{\partial t^{2}} \varphi_{1}^{(2)} \\
(\mu+\kappa) \frac{\partial^{2}}{\partial x_{1}^{2}} u_{2}^{(1)}+\left(\mu_{0}+\kappa_{0}\right) \frac{\partial^{2}}{\partial x_{1}^{2}} u_{2}^{(2)}-\tau u_{2}^{(1)}+\tau u_{2}^{(2)}-\alpha_{13} \frac{\partial}{\partial x_{1}} \varphi_{3}^{(1)}-\alpha_{14} \frac{\partial}{\partial x_{1}} \varphi_{3}^{(2)}=\rho^{(1)} \frac{\partial^{2}}{\partial t^{2}} u_{2}^{(1)} \\
\left(\mu_{0}+\kappa_{0}\right) \frac{\partial^{2}}{\partial x_{1}^{2}} u_{2}^{(1)}+(\bar{\mu}+\bar{\kappa}) \frac{\partial^{2}}{\partial x_{1}^{2}} u_{2}^{(2)}+\tau u_{2}^{(1)}-\tau u_{2}^{(2)}-\alpha_{21} \frac{\partial}{\partial x_{1}} \varphi_{3}^{(1)}-\alpha_{22} \frac{\partial}{\partial x_{1}} \varphi_{3}^{(2)}=\rho^{(2)} \frac{\partial^{2}}{\partial t^{2}} u_{2}^{(2)} \\
\beta \frac{\partial^{2}}{\partial x_{1}^{2}} \varphi_{3}^{(1)}+\beta_{0} \frac{\partial^{2}}{\partial x_{1}^{2}} \varphi_{3}^{(2)}+\alpha_{33} \frac{\partial}{\partial x_{1}} u_{2}^{(1)}-\alpha_{34} \frac{\partial}{\partial x_{1}} u_{2}^{(2)}-\alpha_{35} \varphi_{3}^{(1)}-\alpha_{36} \varphi_{3}^{(2)}=J^{(1)} \frac{\partial^{2}}{\partial t^{2}} \varphi_{3}^{(1)} \\
\beta_{0} \frac{\partial^{2}}{\partial x_{1}^{2}} \varphi_{3}^{(1)}+\bar{\beta} \frac{\partial^{2}}{\partial x_{1}^{2}} \varphi_{3}^{(2)}+\alpha_{41} \frac{\partial}{\partial x_{1}} u_{2}^{(1)}+\alpha_{42} \frac{\partial}{\partial x_{1}} u_{2}^{(2)}-\alpha_{43} \varphi_{3}^{(1)}-\alpha_{44} \varphi_{3}^{(2)}=J^{(2)} \frac{\partial^{2}}{\partial t^{2}} \varphi_{3}^{(2)}
\end{gathered}
$$

and

$$
\begin{aligned}
& (\mu+\kappa) \frac{\partial^{2}}{\partial x_{1}^{2}} u_{3}^{(1)}+\left(\mu_{0}+\kappa_{0}\right) \frac{\partial^{2}}{\partial x_{1}^{2}} u_{3}^{(2)}-\tau u_{3}^{(1)}+\tau u_{3}^{(2)}+\alpha_{13} \frac{\partial}{\partial x_{1}} \varphi_{2}^{(1)}+\alpha_{14} \frac{\partial}{\partial x_{1}} \varphi_{2}^{(2)}=\rho^{(1)} \frac{\partial^{2}}{\partial t^{2}} u_{3}^{(1)} \\
& \left(\mu_{0}+\kappa_{0}\right) \frac{\partial^{2}}{\partial x_{1}^{2}} u_{3}^{(1)}+(\bar{\mu}+\bar{\kappa}) \frac{\partial^{2}}{\partial x_{1}^{2}} u_{3}^{(2)}+\tau u_{3}^{(1)}-\tau u_{3}^{(2)}+\alpha_{21} \frac{\partial}{\partial x_{1}} \varphi_{2}^{(1)}+\alpha_{22} \frac{\partial}{\partial x_{1}} \varphi_{2}^{(2)}=\rho^{(2)} \frac{\partial^{2}}{\partial t^{2}} u_{3}^{(2)} \\
& \beta \frac{\partial^{2}}{\partial x_{1}^{2}} \varphi_{2}^{(1)}+\beta_{0} \frac{\partial^{2}}{\partial x_{1}^{2}} \varphi_{2}^{(2)}-\alpha_{33} \frac{\partial}{\partial x_{1}} u_{3}^{(1)}+\alpha_{34} \frac{\partial}{\partial x_{1}} u_{3}^{(2)}-\alpha_{35} \varphi_{2}^{(1)}-\alpha_{36} \varphi_{2}^{(2)}=J^{(1)} \frac{\partial^{2}}{\partial t^{2}} \varphi_{2}^{(1)} \\
& \beta_{0} \frac{\partial^{2}}{\partial x_{1}^{2}} \varphi_{2}^{(1)}+\bar{\beta} \frac{\partial^{2}}{\partial x_{1}^{2}} \varphi_{2}^{(2)}-\alpha_{41} \frac{\partial}{\partial x_{1}} u_{3}^{(1)}-\alpha_{42} \frac{\partial}{\partial x_{1}} u_{3}^{(2)}-\alpha_{43} \varphi_{2}^{(1)}-\alpha_{44} \varphi_{2}^{(2)}=J^{(2)} \frac{\partial^{2}}{\partial t^{2}} \varphi_{2}^{(2)}
\end{aligned}
$$

The components $u_{1}^{(1)}$ and $u_{1}^{(2)}$ describe the longitudinal displacement waves, while $\varphi_{1}^{(1)}$ and $\varphi_{1}^{(2)}$ describe the longitudinal microrotation waves. On the other hand the components $u_{2}^{(1)}, u_{2}^{(2)}, u_{3}^{(1)}$ and $u_{3}^{(2)}$ describe the transverse displacement waves, while $\varphi_{2}^{(1)}, \varphi_{2}^{(2)}, \varphi_{3}^{(1)}$ and $\varphi_{3}^{(2)}$ describe the transverse microrotation waves.

From the above equations, we can remark that the longitudinal waves and the transverse waves are separable. Moreover, the longitudinal displacement waves and the longitudinal microrotation waves are also separable.

In the Twiss-Eringen [5] theory, constructed by using the Eulerian description, the longitudinal microrotation waves of one constituent are not influenced by the longitudinal microrotation waves of the other constituent. In the theory considered here, the longitudinal microrotation waves of the two constituents are coupled. Moreover, as we will see below, the coupling coefficients for both the longitudinal displacement waves and the longitudinal microrotation waves are more complex and depend on frequency. 
Let us consider first the equations $(5.1)_{1}$ and $(5.1)_{2}$. We seek solutions of the following form

$$
u_{1}^{(\sigma)}=\operatorname{Re}\left\{A_{1}^{(\sigma)} \exp \left[i\left(\varkappa x_{1}-\omega t\right)\right]\right\}
$$

where $i=\sqrt{-1}, A_{1}^{(1)}$ and $A_{1}^{(2)}$ denote the amplitudes, $\omega$ is a real positive constant, $\varkappa$ is a complex number, $c=\frac{\omega}{\varkappa}$ is the speed of propagation, $\frac{2 \pi}{\operatorname{Re}[\varkappa]}$ is the wavelength and $\operatorname{Re}\left[\frac{\omega}{2 \pi}\right]$ is the frequency. $\operatorname{If} \operatorname{Im}[c] \neq 0$ then the waves are damped, whereas if $c$ is a real number the waves are undamped.

The amplitudes $A_{1}^{(\sigma)}$ are solutions of the following algebraic system

$$
\begin{aligned}
& \left(c_{11} \frac{\omega^{2}}{c^{2}}+\tau-\rho^{(1)} \omega^{2}\right) A_{1}^{(1)}+\left(c_{10} \frac{\omega^{2}}{c^{2}}-\tau\right) A_{1}^{(2)}=0 \\
& \left(c_{10} \frac{\omega^{2}}{c^{2}}-\tau\right) A_{1}^{(1)}+\left(c_{12} \frac{\omega^{2}}{c^{2}}+\tau-\rho^{(2)} \omega^{2}\right) A_{1}^{(2)}=0
\end{aligned}
$$

where

$$
\begin{aligned}
& c_{11}=\lambda+2 \mu+\kappa \\
& c_{10}=\lambda_{0}+2 \mu_{0}+\kappa_{0} \\
& c_{12}=\bar{\lambda}+2 \bar{\mu}+\bar{\kappa}
\end{aligned}
$$

and $c$ is solution of the following equation

$$
c^{4}\left(\tau \rho^{(1)}+\tau \rho^{(2)}-\omega^{2} \rho^{(1)} \rho^{(2)}\right)-c^{2}\left(2 \tau c_{10}+\tau c_{11}+\tau c_{12}-\omega^{2} c_{12} \rho^{(1)}-\omega^{2} c_{11} \rho^{(2)}\right)+\omega^{2} c_{10}^{2}-\omega^{2} c_{11} c_{12}=0
$$

Let us analyze this algebraic equation. We note that the positive definiteness condition on the internal energy density implies that

$$
c_{11} c_{12}>c_{10}^{2}, \quad \tau>0
$$

First, we consider

$$
\omega^{2} \neq \frac{\tau\left(\rho^{(1)}+\rho^{(2)}\right)}{\rho^{(1)} \rho^{(2)}}
$$

In this case, the speed is given by the relations

$$
c_{ \pm}^{2}=\frac{1}{2\left[\tau\left(\rho^{(2)}+\rho^{(1)}\right)-\omega^{2} \rho^{(1)} \rho^{(2)}\right]}\left[\tau\left(2 c_{10}+c_{11}+c_{12}\right)-\omega^{2}\left(c_{12} \rho^{(1)}+c_{11} \rho^{(2)}\right) \pm \sqrt{\Delta}\right]
$$

where

$$
\Delta=\left[2 \tau c_{10}+c_{12}\left(\tau-\omega^{2} \rho^{(1)}\right)+c_{11}\left(\tau-\omega^{2} \rho^{(2)}\right)\right]^{2}+4 \omega^{2}\left(c_{11} c_{12}-c_{10}^{2}\right)\left[\tau\left(\rho^{(2)}+\rho^{(1)}\right)-\omega^{2} \rho^{(1)} \rho^{(2)}\right]
$$

and the subscripts \pm corresponds to the \pm from the right side. In order to study the nature of these roots, let us remark that $\Delta$ can be written as

$$
\begin{aligned}
\Delta= & \tau^{2}\left(2 c_{10}+c_{11}+c_{12}\right)^{2}-\omega^{2} 2 \tau\left\{\left[\left(c_{10}+c_{12}\right)^{2}+\left(c_{10}^{2}-c_{11} c_{12}\right)\right] \rho^{(1)}+\left[\left(c_{10}+c_{11}\right)^{2}+\left(c_{10}^{2}-c_{11} c_{12}\right)\right] \rho^{(2)}\right\} \\
& +\omega^{4}\left[4 c_{10}^{2} \rho^{(1)} \rho^{(2)}+\left(c_{12} \rho^{(1)}-c_{11} \rho^{(2)}\right)^{2}\right]
\end{aligned}
$$

So, viewing $\Delta$ as a second degree expression in $\omega^{2}$, then the discriminant of the equation $\Delta=0$ is

$$
\delta=\left(c_{10}^{2}-c_{11} c_{12}\right)\left(\tau\left(c_{10}+c_{12}\right) \rho^{(1)}-\tau\left(c_{10}+c_{11}\right) \rho^{(2)}\right)^{2}<0
$$

and, in view of the inequality (5.8), we deduce that $\Delta>0$ for all values of $\omega$. Thus, the quantities given by (5.10) are well defined. 
If

$$
\omega^{2}<\frac{\tau\left(\rho^{(1)}+\rho^{(2)}\right)}{\rho^{(1)} \rho^{(2)}}
$$

then, in view of (5.8), it follows that $c_{+}^{2}>0$ and $c_{-}^{2}<0$. So, we have two possible real speeds and two possible purely imaginary speeds. However, one of the purely imaginary speeds, that is the one for which $\operatorname{Im}[c]<0$, leads to harmonic vibrations whose amplitudes increase exponentially with distance, which is an unacceptable physically phenomenon. In conclusion, there are possible one damped wave and two undamped waves.

If

$$
\omega^{2}>\frac{\tau\left(\rho^{(1)}+\rho^{(2)}\right)}{\rho^{(1)} \rho^{(2)}}
$$

then from (5.8), (5.10) and (5.11) it follows that

$$
\operatorname{sign}\left[c_{ \pm}^{2}\right]=\operatorname{sign}\left[-\tau\left(2 c_{10}+c_{11}+c_{12}\right)+\omega^{2}\left(c_{12} \rho^{(1)}+c_{11} \rho^{(2)}\right)\right]
$$

But, in view of the arithmetic-geometric inequality and the inequalities (5.8), we deduce that

$$
\frac{\tau\left(\rho^{(1)}+\rho^{(2)}\right)}{\rho^{(1)} \rho^{(2)}}>\frac{\tau\left(2 c_{10}+c_{11}+c_{12}\right)}{c_{12} \rho^{(1)}+c_{11} \rho^{(2)}}
$$

and thus

$$
c_{ \pm}^{2}>0
$$

In conclusion, all the possible waves are undamped in the case (5.15).

To finish the discussion, we note that for

$$
\omega^{2}=\frac{\tau\left(\rho^{(1)}+\rho^{(2)}\right)}{\rho^{(1)} \rho^{(2)}}
$$

from (5.7) we obtain

$$
c= \pm \sqrt{\frac{\left(c_{11} c_{12}-c_{10}^{2}\right)\left(\rho^{(1)}+\rho^{(2)}\right)}{\left(\rho^{(1)}\right)^{2} c_{12}+\left(\rho^{(2)}\right)^{2} c_{11}-2 c_{10} \rho^{(1)} \rho^{(2)}}}
$$

Now, let us consider the equations $(5.1)_{3}$ and $(5.1)_{4}$. As above, we seek solutions of the form

$$
\varphi_{1}^{(\sigma)}=\operatorname{Re}\left\{B_{1}^{(\sigma)} \exp \left[i\left(\wp x_{1}-\omega t\right)\right]\right\}
$$

For the amplitudes $B_{1}^{(1)}, B_{1}^{(2)}$ we find

$$
\begin{aligned}
& \left(c_{31} \frac{\omega^{2}}{h^{2}}+\alpha_{35}-J^{(1)} \omega^{2}\right) B_{1}^{(1)}+\left(c_{30} \frac{\omega^{2}}{h^{2}}+\alpha_{36}\right) B_{1}^{(2)}=0 \\
& \left(c_{30} \frac{\omega^{2}}{h^{2}}+\alpha_{43}\right) B_{1}^{(1)}+\left(c_{32} \frac{\omega^{2}}{h^{2}}+\alpha_{44}-J^{(2)} \omega^{2}\right) B_{1}^{(2)}=0
\end{aligned}
$$

where

$$
\begin{aligned}
& c_{31}=\alpha+\beta+\gamma \\
& c_{30}=\alpha_{0}+\beta_{0}+\gamma_{0} \\
& c_{32}=\bar{\alpha}+\bar{\beta}+\bar{\gamma}
\end{aligned}
$$


and $h=\frac{\omega}{\wp}$ is solution of the following equation

$$
\begin{aligned}
& h^{4}\left[\omega^{4} J^{(1)} J^{(2)}-\omega^{2}\left(J^{(2)} \alpha_{35}+\omega^{2} J^{(1)} \alpha_{44}\right)-\alpha_{36} \alpha_{43}+\alpha_{35} \alpha_{44}\right] \\
& +h^{2}\left[-\omega^{4}\left(c_{32} J^{(1)}+c_{31} J^{(2)}\right)+\omega^{2}\left(-c_{30} \alpha_{36}-c_{30} \alpha_{43}+c_{31} \alpha_{44}+c_{32} \alpha_{35}\right)\right]-\omega^{4}\left(c_{30}^{2}-c_{31} c_{32}\right)=0
\end{aligned}
$$

The solutions of the above equation are given by

$$
h_{ \pm}^{2}=\omega^{2} \frac{c_{32}\left(\omega^{2} J^{(1)}-\alpha_{35}\right)+c_{30}\left(\alpha_{36}+\alpha_{43}\right)+c_{31}\left(\omega^{2} J^{(2)}-\alpha_{44}\right) \pm \sqrt{\widetilde{\Delta}}}{2\left(-\alpha_{36} \alpha_{43}+\left(\omega^{2} J^{(1)}-\alpha_{35}\right)\left(\omega^{2} J^{(2)}-\alpha_{44}\right)\right)}
$$

where

$$
\begin{aligned}
\widetilde{\Delta}= & {\left[c_{32}\left(\omega^{2} J^{(1)}-\alpha_{35}\right)+c_{30}\left(\alpha_{36}+\alpha_{43}\right)+c_{31}\left(\omega^{2} J^{(2)}-\alpha_{44}\right)\right]^{2} } \\
& +4\left(c_{30}^{2}-c_{31} c_{32}\right)\left[-\alpha_{36} \alpha_{43}+\left(\omega^{2} J^{(1)}-\alpha_{35}\right)\left(\omega^{2} J^{(2)}-\alpha_{44}\right)\right]
\end{aligned}
$$

The positive definiteness condition on the internal energy density implies that

$$
c_{31}>0, \quad c_{31} c_{32}>c_{30}^{2}
$$

and

$$
\alpha_{35} \alpha_{44}>\alpha_{36} \alpha_{43}
$$

In the following, we identify frequencies for which we have undamped longitudinal microrotation waves. The study is motivated by the results obtained for one micropolar constituent [14], where some conditions have been imposed on the frequency in order to have undamped longitudinal waves.

To this aim, we split our study in two cases: when $c_{30} \neq 0$ and when $c_{30}=0$, respectively.

i) $c_{30} \neq 0$

Let us consider the quantity

$$
\tilde{\delta}=\left(J^{(2)} \alpha_{35}-J^{(1)} \alpha_{44}\right)^{2}+4 J^{(1)} J^{(2)} \alpha_{36} \alpha_{43}
$$

We define the frequencies

$$
\begin{aligned}
& \omega_{1}^{2}= \begin{cases}\max \left\{\frac{J^{(2)} \alpha_{35}+J^{(1)} \alpha_{44}+\sqrt{\tilde{\delta}}}{2 J^{(1)} J^{(2)}}, 0\right\}, & \text { if } \quad \tilde{\delta} \geq 0 \\
0, & \text { if } \quad \tilde{\delta}<0\end{cases} \\
& \omega_{2}^{2}=\max \left\{\frac{\alpha_{35}}{J^{(1)}}-\frac{c_{31} \alpha_{36}}{c_{30} J^{(1)}}, \frac{\alpha_{44}}{J^{(2)}}-\frac{c_{32} \alpha_{43}}{c_{30} J^{(2)}}, 0\right\} \\
& \omega_{3}^{2}=\max \left\{\frac{\alpha_{35}}{J^{(1)}}-\frac{c_{30} \alpha_{36}}{c_{32} J^{(1)}}, \frac{\alpha_{44}}{J^{(2)}}-\frac{c_{30} \alpha_{43}}{c_{31} J^{(2)}}, 0\right\}
\end{aligned}
$$

Let us remark that $\widetilde{\Delta}$ can be written in the following form

$$
\begin{aligned}
\widetilde{\Delta}= & {\left[c_{32}\left(\omega^{2} J^{(1)}-\alpha_{35}\right)-c_{31}\left(\omega^{2} J^{(2)}-\alpha_{44}\right)+c_{30}\left(\alpha_{36}-\alpha_{43}\right)\right]^{2} } \\
& +4\left[c_{30}\left(\omega^{2} J^{(1)}-\alpha_{35}\right)+c_{31} \alpha_{36}\right]\left[c_{30}\left(\omega^{2} J^{(2)}-\alpha_{44}\right)+c_{32} \alpha_{43}\right]
\end{aligned}
$$

It is easy to see from (5.31) that the condition

$$
\omega^{2} \geq \omega_{2}^{2}
$$


is sufficient to have $\widetilde{\Delta} \geq 0$.

On the other hand, if we consider the frequencies for which

$$
\omega^{2} \geq \omega_{1}^{2}
$$

then

$$
-\alpha_{36} \alpha_{43}+\left(\omega^{2} J^{(1)}-\alpha_{35}\right)\left(\omega^{2} J^{(2)}-\alpha_{44}\right) \geq 0
$$

Hence, we can see that if the frequency is such that

$$
\omega^{2} \geq \max \left\{\omega_{1}^{2}, \omega_{2}^{2}, \omega_{3}^{2}\right\}
$$

then $h_{ \pm}$are real numbers, so the corresponding longitudinal microrotation waves are undamped.

ii) $c_{30}=0$

For this case, we restrict our analysis to the mixtures for which

$$
\alpha_{36} \alpha_{43} \geq 0
$$

In view of the assumption (5.36) we have that $\tilde{\delta} \geq 0$. Moreover, in view of (5.31) we have that $\tilde{\Delta}>0$ for all frequencies.

In conclusion, the corresponding longitudinal microrotation waves are undamped for the frequencies which satisfy

$$
\omega^{2} \geq \max \left\{\frac{\alpha_{35}}{J^{(1)}}, \frac{\alpha_{44}}{J^{(2)}}, \omega_{1}^{2}\right\}
$$

Let us remark that (5.35) and (5.37) are generalizations of the condition imposed in the case of one constituent [14].

The analysis concerning the speeds of propagation for transverse displacement waves and transverse microrotation waves involves more complicated computations, since the corresponding equations are coupled. Numerical calculus for particular types of mixtures are more appropriated.

\section{ACKNOWLEDGEMENT}

The authors acknowledge support from the Romanian Ministry of Education and Research, CNCSIS Grant code TE-184, no. 86/30.07.2010.

\section{References}

[1] R. M. Bowen, Theory of mixtures, Continuum physics, vol. III, (in A.C. Eringen ed.), Academic Press, New York, 1976.

[2] A. Bedford and D.S. Drumheller, Theories of immiscible and structured mixtures, Int. J. Engng. Sci., vol. 21, pp. 863-960, 1983.

[3] K. R. Rajagopal and L. Tao, Mechanics of mixtures. Series on Advances in Mathematics for Applied Science, vol. 35, World Scientific, Singapore, 1995.

[4] R. J. Twiss and A. C. Eringen, Theory of mixtures for micromorphic materials-I, Int. J. Engng. Sci., vol. 9, pp. 1019-1044, 1971.

[5] R. J. Twiss and A. C. Eringen, Theory of mixtures for micromorphic materials. II. Elastic constitutive equations, Int. J. Engng. Sci., vol. 10, pp. 437-465, 1972.

[6] A. C. Eringen, Micropolar mixture theory of porous media, Journal of Applied Physics, vol. 94, pp. 41844190, 2003. 
[7] A. C. Eringen, A mixture theory for geophysical fluids, Nonlinear Process in Geophysics, vol. 11, pp. 75-82, 2004 .

[8] D. Ieşan, A theory of thermoviscoelastic composites modelled as interacting Cosserat continua, J. Thermal Stresses, vol. 30, pp. 1269-1289, 2007.

[9] C. Galeş, A mixture theory for micropolar thermoelastic solids, Mathematical Problems in Engineering, Vol. 2007, Article ID 90672, 21 pages, 2007.

[10] S. Chiriţă and C. Galeş, A mixture theory for microstretch thermoviscoelastic solids, J. Thermal Stresses, vol. 31, pp. 1099-1124, 2008.

[11] D. Ieşan, On the Theory of Mixtures of Thermoelastic Solids, J. Thermal Stresses, vol. 14, pp. 389-408, 1991.

[12] D. Ieşan, On the theory of viscoelastic mixtures, J. Thermal Stresses, vol. 27, pp. 1125-1148, 2004.

[13] A. C. Eringen, Microcontinuum field theories. Foundations and solids, Springer, New York, 1999.

[14] D. Ieşan and M. Ciarletta, Non-classical elastic solids, Longman Scientific and Technical, Harlow, Essex, UK and John Wiley\&Sons, Inc., New York, 1993.

[15] F. Martínes and R. Quintanilla, Some qualitative results for the linear theory of binary mixtures of thermoelastic solids, Collect. Math., vol. 46, pp. 263-277, 1995.

[16] C. Galeş, Some results in the dynamics of viscoelastic mixtures, Mathematics and Mechanics of Solids, vol. 13, pp. 124-147, 2008.

[17] R. A. Adams, Sobolev Spaces, Academic Press, New York, 1975.

[18] V. Barbu, Partial differential equations and boundary value problems, Kluwer Academic Publishers, 1998.

[19] S. Chiriţă, Uniqueness and continuous data dependence of solutions to the incompressible micropolar flows forward and backward in time, Int. J. Engng. Sci., vol. 39, pp. 1787-1802, 2001.

[20] A. Pazy, Semigroups of linear operators and applications to partial differential equations, Springer Verlag, New York, Berlin, Heidelberg, Tokyo, 1983.

[21] I. Vrabie, $C_{0}$-Semigroups and applications, Ser. Mathematics Studies no. 191, Elsevier, North-Holland Amsterdam, 2003. 